\title{
Synthesis, characterization and electrical properties of nano metal and metal-oxide doped with conducting polymer composites by in-situ chemical polymerization
}

\begin{abstract}
The conducting polyaniline (pani) doped with nano aluminium (n-Alpani) and nano aluminium oxide ( $\mathrm{n}-\mathrm{Al}_{2} \mathrm{O}_{3}$ pani) composites are prepared by using in situ chemical oxidative polymerization method. The synthesized samples were characterized Fourier transform infrared spectroscopy (FT-IR), X-ray diffraction (XRD). The morphological and elemental compositions were analyzed using FESEM with EDX. The electrical conductivity of the pure Pani and its nanocomposites were studied by two probe technique. The very strong bands at $593 \mathrm{~cm}^{-1}$ and $615 \mathrm{~cm}^{-1}$ corresponds to Al-O stretching vibrations. FT-IR study confirms the presence of aluminium nanoparticles in the Pani matrix. The A.C electrical conductivity of Pani, $\mathrm{n}-\mathrm{Al}_{2} \mathrm{O}_{3}$ pani and $\mathrm{n}-\mathrm{Al}$ pani were calculated and found to be $8.2 \times 10^{-}$ ${ }^{9} \mathrm{~S} / \mathrm{cm}, 1.77 \times 10^{-8} \mathrm{~S} / \mathrm{cm}$ and $2.15 \times 10^{-6} \mathrm{~S} / \mathrm{cm}$ respectively. The results revealed that there is an increase of one order of conductivity in the metal oxide composite and three order increase of conductivity in the nano metal composite when compared to pure polyaniline.
\end{abstract}

Volume I Issue 6 - 2017

\author{
Subramanian Senthilkumar, Annamalai \\ Rajendran \\ Department of Physics, Nehru Memorial College, India
}

Correspondence: Annamalai Rajendran, Department of Physics, Nehru Memorial College (Autonomous), Puthanampatti, Tiruchirappalli, Tamilnadu, India -621007,

Email neelrajnmc@gmail.com

Received: November 06, 2017 | Published: December 07, 2017

Keywords: polyaniline, FT-IR, XRD, FESEM(EDX), electrical conductivity

Abbreviations: FESEM, field emission scanning electron microscope; PANI, polyaniline; n-Alpani, nano aluminium; XRD, $\mathrm{x}$-ray diffraction; NCs, nanocomposites

\section{Introduction}

In recent years, developments of inorganic-organic hybrid materials on nanometer scale have been receiving significant attention due to a wide range of potential applications and high absorption in the visible part of the spectrum and high mobility of the charge carriers. ${ }^{1-3}$ Conductive polymer with polyaromatic backbone including polypyrrole, polythiophene, polyaniline, etc. The received a great deal of attention in the last two decades. ${ }^{4}$ Amongst the family of conducting polymers polyaniline (PANI) is one of the most promising electrically conducting polymers due to its unique electrical, electrochemical properties, easy polymerization, high environmental stability and low cost of monomer.

A number of metal and metal oxide particles have been encapsulated into the conductive polymer to form nanocomposites (NCs). The NCs exhibit combination of properties like conductivity, electrochemical, catalytic and optical properties. The NCs are used in applications like electro chromic devices, light-emitting diodes, electromagnetic interference shielding, secondary batteries, electrostatic discharge systems, chemical and bio-chemical sensors. ${ }^{5}$ The present paper reports the synthesis of polyaniline nanocomposite by the incorporation of $\mathrm{n}-\mathrm{Al}$ and $\mathrm{n}-\mathrm{Al}_{2} \mathrm{O}_{3}$ particles in the polyaniline matrix. To achieve this goal, pani, n-Alpani and $\mathrm{n}-\mathrm{Al}_{2} \mathrm{O}_{3}$ pani have been synthesized by in-situ chemical oxidation method. These polymer hybrid nanocomposites have been characterized using various techniques. The structural properties were analyzed by FT-IR and XRD. The interaction of $\mathrm{n}-\mathrm{Al}$ and $\mathrm{n}-\mathrm{Al}_{2} \mathrm{O}_{3}$ composite with the polyaniline has been visualized using the Field Emission Scanning Electron Microscope (FESEM). The electrical conductivity measurement was studied using two probe techniques.

\section{Experimental details}

\section{Characterization Techniques}

FT-IR spectra were recorded on a Bruker Alpha T FT-IR spectrometer. IR spectra of the samples were recorded at room temperature in the mid IR region of $4000-400 \mathrm{~cm}^{-1}$. The XRD pattern was recorded using $\mathrm{CuK} \alpha$ radiation $\left(\lambda=1.54060 \mathrm{~A}^{\circ}\right)$ with nickel monochromatic in the range of $2 \Theta$ from $10^{\circ}$ to $80^{\circ}$. The FESEMEDX was recorded using JEOL-Model 6390 machine. Conductivity measurements were performed by a typical Two Probe method with PSM 1735 Frequency Response Analyzer employing the pressed pellet method over the frequency range from $1 \mathrm{KHz}$ to $10 \mathrm{MHz}$ at room temperature. Spectrum of visible light is measured using absorption spectrometer of Stellar Net Inc (model EPP2000). The power of visible light is measured using Newport optical power meter (model 1916-R) and is found to be $600 \mathrm{~mW}$ at wavelength of $650 \mathrm{~nm}$ (maximum intensity of the light spectrum).

\section{Synthesis of polyaniline}

$0.1 \mathrm{M}$ of aniline was dissolved in $100 \mathrm{ml}$ of de-ionized water and stirred for $15 \mathrm{~min}$ using a magnetic stirrer. $1 \mathrm{M}$ of $\mathrm{H}_{2} \mathrm{SO}_{4}$ was added slowly from drop to the aniline monomer solution. $0.1 \mathrm{M}$ of ammonium per sulphate was dissolved in $20 \mathrm{ml}$ of deionized water and slowly added drop by drop for half an hour from a burette vertically to the above prepared solution. After stirring for $5 \mathrm{~h}$, the solution was filtered and the residual was washed with double distilled water, methanol and acetone, and then dried in an oven at $60^{\circ} \mathrm{C}$. The final product was grounded into a fine powder. 
Preparation of $\mathrm{Al}$ and $\mathrm{Al}_{2} \mathrm{O}_{3}$ doped with pani nanocomposites: To synthesize $\mathrm{n}-\mathrm{Al}$ pani composite, $0.1 \mathrm{M}$ aniline monomer and $1 \mathrm{M}$ $\mathrm{H}_{2} \mathrm{SO}_{4}$ were stirred with double distilled water, and the required quantity $(40 \%)$ of nano aluminium (Al) powder was added. The oxidant APS was added drop-wise to the aniline-acid-aluminium (Al) mixture with constant stirring. The reaction was conducted at room temperature. When the sample was reacted in the mixed solution of aniline- $\mathrm{NH}_{4} \mathrm{~S}_{2} \mathrm{O}_{8}-\mathrm{H}_{2} \mathrm{SO}_{4}$, the color of the sample changed to light blue, revealing formation of PANI through an oxidation reaction. The stirring was continued for $5 \mathrm{~h}$ to ensure complete polymerization. A dark green n-Alpani nanocomposite was thus formed, followed by a color change to dark blue. The composite obtained was filtered and washed with distilled water and methanol to remove excess acid. The product was dried in an oven at $60^{\circ} \mathrm{C}$ for $12 \mathrm{~h}$. The same process was continued with $40 \%$ weight of nano $\mathrm{Al}_{2} \mathrm{O}_{3}$ with respect to aniline monomer. The dried n-Alpani and $\mathrm{n}-\mathrm{Al}_{2} \mathrm{O}_{3}$ pani composite was fineground using a mortar.

\section{Results and discussion}

\section{FT-IR analysis}

The FT-IR spectroscopy study of pure Pani, n- $\mathrm{Al}_{2} \mathrm{O}_{3}$ pani and n-Alpani nanocomposites are shown in Figure 1 (A-C). From Figure $1 \mathrm{~A}$, the very strong characteristic peak at $3741 \mathrm{~cm}^{-1}$ is assigned to the N-H stretching vibration of amino group of polyaniline. ${ }^{6}$ The absorption band at $2361 \mathrm{~cm}^{-1}$ are correspond to $v(\mathrm{~N}-\mathrm{H})+$ unsaturated amine. The very strong peak at $1504 \mathrm{~cm}^{-1}$ is associated with N-B-N stretching vibrations (where $\mathrm{B}$ refers to benzenoid ring). The peaks observed in the present work well matches with Pani ${ }^{5-10}$ Figure 1B \& Figure 1C) shows all the characteristics peak of Pani with some slight spectral intensity changes. The strong peaks at $2926 \mathrm{~cm}^{-1}$ are assigned to asymmetric and symmetric stretching vibration mode of methyl groups. ${ }^{7}$ The absorption band at $1296 \mathrm{~cm}^{-1}$ was assigned to C-N stretching of secondary aromatic amine. The characteristic absorption bands at $1151 \mathrm{~cm}^{-1}$ are corresponds to $(\mathrm{C}=\mathrm{N})$ stretching vibration. ${ }^{8}$ The characteristic peak at $1114 \mathrm{~cm}^{-1}$ are due to in-plane bending vibration of $\mathrm{C}-\mathrm{H}$ mode. ${ }^{9-11} \mathrm{~A}$ broad and smooth absorption band in the wave number range from about $400-900 \mathrm{~cm}^{-1}$ reveals the formation of $\mathrm{Al}-\mathrm{O}$ vibrations. The polymerization was confirmed by FTIR spectroscopy. Two additional bands appeared at $615 \mathrm{~cm}^{-1}$ and $593 \mathrm{~cm}^{-1}$ were assigned to Al-O stretching vibrations. This confirms the interaction of $\mathrm{n}-\mathrm{Al}$ and $\mathrm{n}-\mathrm{Al}_{2} \mathrm{O}_{3}$ nano particles in the conducting polymer matrix. ${ }^{12-14}$

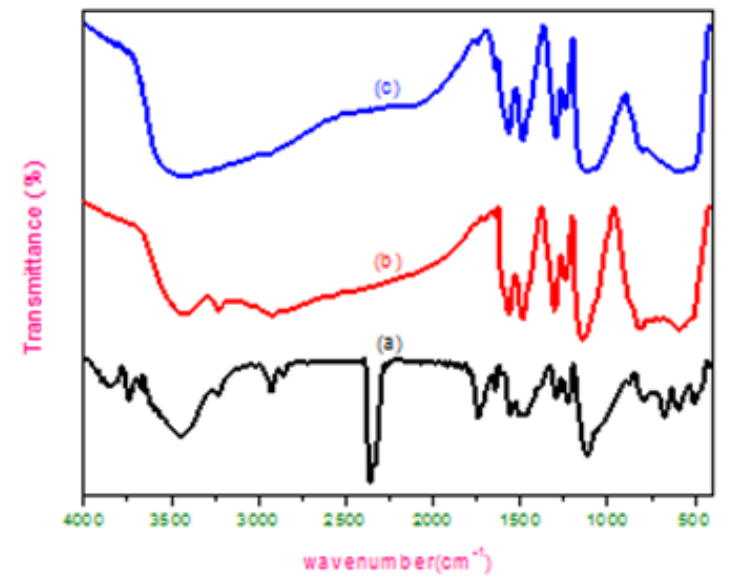

Figure I FT- IR spectrum of (A) Pure polyaniline (B) $n-\mathrm{Al}_{2} \mathrm{O}_{3}$ Pani (C) n-AIPani.

\section{XRD analysis}

The X-Ray diffraction patterns of the Pani, $\mathrm{n}-\mathrm{Al}_{2} \mathrm{O}_{3}$ pani and n-Alpani nanocomposites are shown in Figure 2 (A-C) respectively. XRD studies showed that Pani is amorphous in nature which shown in the Figure $2(A)$. The broad diffraction peak at $2 \theta=24^{\circ}$ is characteristic peak for Pani. ${ }^{15}$

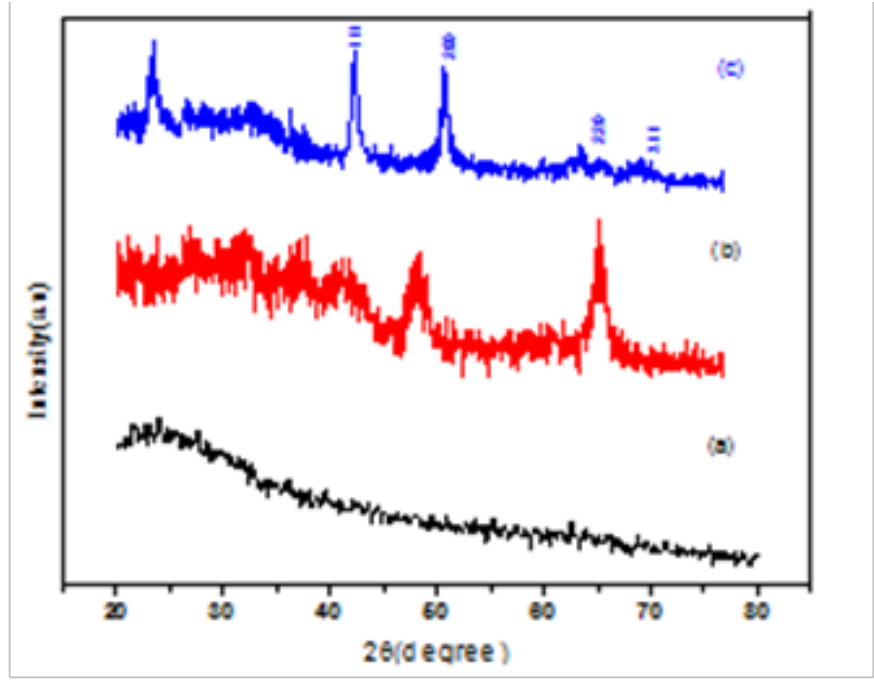

Figure 2 XRD spectra of (A) Pure polyaniline (B) $n-\mathrm{Al}_{2} \mathrm{O}_{3}$ Pani (C) n-AlPani.

$\mathrm{XRD}$ pattern of $\mathrm{n}-\mathrm{Al}_{2} \mathrm{O}_{3}$ pani and n-Alpani are shown in the Figure $2 \mathrm{~B} \&$ Figure $2 \mathrm{C}$ ). After doping the samples showed crystalline nature which was confirmed by the peaks at about $2 \theta=37.81^{\circ}, 48.28^{\circ}, 64.32^{\circ}$ and $70.81^{\circ}$ for n-Alpani and $2 \theta=36.32^{\circ}, 45.12^{\circ}$ and $66.83^{\circ}$ for $\mathrm{n}-\mathrm{Al}_{2} \mathrm{O}_{3}$ pani. These peaks were matched with JCPDS data of Aluminium file no. 04-0787 and Aluminium oxide JCPDS card no. 79-1557. The planes corresponding to n-Alpani is (111), (220), (200), (311) and for $\mathrm{n}-\mathrm{Al}_{2} \mathrm{O}_{3}$ pani corresponds to (440), (400) and (311). The XRD patterns of $\mathrm{n}-\mathrm{Al}_{2} \mathrm{O}_{3}$ pani and $\mathrm{n}$-Alpani nanocomposites are compared in Figure 2(A). The peak shows sharp and well-defined, indicating the crystallinity of the synthesized materials. The average crystallite sizes have been estimated to be around n-Alpani and $n-\mathrm{Al}_{2} \mathrm{O}_{3}$ pani is 31 and $38 \mathrm{~nm}$ respectively.

\section{FE-SEM analysis}

FESEM was performed in order to investigate surface morphology of the polymers and the nanocomposites. EDAX was done to reveal the chemical composition of the samples. Figure 3A-F shows the SEM and EDAX images of Pani, n- $\mathrm{Al}_{2} \mathrm{O}_{3}$ Pani and n-Alpani with weight percentages are shown in the Tables 1-3. The FESEM image of Pani is spherical and aggregated globules. In the nano composites some of the Aluminium particles seemed to be embedded in the polymer matrix and started coalescing (a tendency to coalesce and form agglomerates) due to the surface absorption property of PANI. The change in morphology can be explained by the absorption and intercalation of PANI on the surface of $\mathrm{Al}_{2} \mathrm{O}_{3}$.

The aniline monomer is likely to be absorbed the surface of $\mathrm{Al}_{2} \mathrm{O}_{3}$ through electrostatic attraction and by the formation of weak chargetransfer complexes between aniline monomer and the structure of $\mathrm{Al}_{2} \mathrm{O}_{3}{ }^{16}$ The EDX result shows that $\mathrm{n}-\mathrm{Al}$ and $\mathrm{n}-\mathrm{Al}_{2} \mathrm{O}_{3}$ was present in the nanocomposite and the weight percentages are shown in Tables (1-3). 


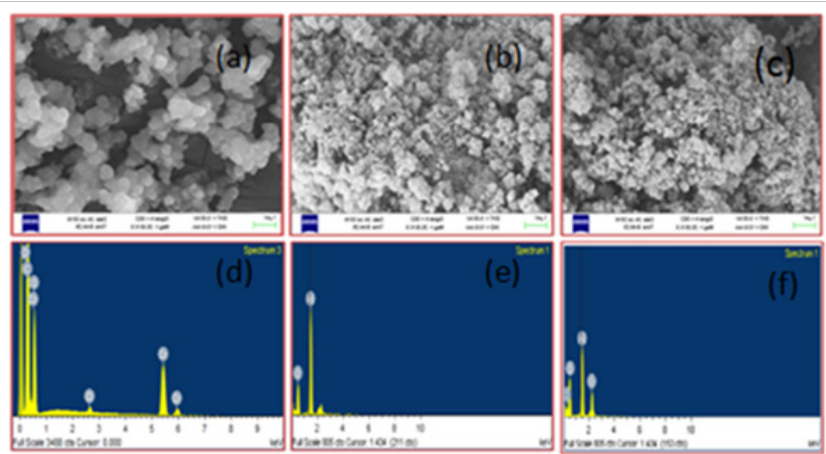

Figure 3 FESEM image of (A) Pani (B) n-Alpani (C) $n-\mathrm{Al}_{2} \mathrm{O}_{3}$ pani and EDX spectra of (D) Pani, (E) n-Alpani (F) n-Al $\mathrm{O}_{3}$ pani.

Table I Elemental concentration for Pani

\begin{tabular}{lll}
\hline Element & Weight\% & Atomic\% \\
\hline C K & 55.27 & 69.41 \\
O K & 26.87 & 25.33 \\
CI K & 0.59 & 0.25 \\
Cr K & 17.27 & 5.01 \\
Total & 100 & 100 \\
\hline
\end{tabular}

Table 2 Elemental concentration for n-AIPani

\begin{tabular}{lll}
\hline Element & Weight\% & Atomic\% \\
\hline Al K & 52.93 & 40 \\
O K & 47.07 & 60 \\
Total & 100 & 10 \\
\hline
\end{tabular}

Table 3 Elemental concentration for $n-n-\mathrm{Al}_{2} \mathrm{O}_{3}$ Pani

\begin{tabular}{lll}
\hline Element & Weight\% & Atomic\% \\
\hline C K & 17.6 & 23.87 \\
Al K & 18.8 & 11.35 \\
O K & 63.6 & 64.77 \\
Total & 100 & 100
\end{tabular}

\section{A. C electrical conductivity studies}

The A. C Conductivity measurements have been performed by a typical two probe technique. The A. C electrical conductivities of Pani, $\mathrm{n}-\mathrm{Al}_{2} \mathrm{O}_{3}$ Pani and n-Alpani are shown in Figure 4. The A.C electrical conductivity of Pani, $\mathrm{n}-\mathrm{Al}_{2} \mathrm{O}_{3}$ pani and $\mathrm{n}$-Alpani were calculated and found to be $8.2 \times 10^{-9} \mathrm{~S} / \mathrm{cm}, 1.77 \times 10^{-8} \mathrm{~S} / \mathrm{cm}$ and $2.15 \times 10^{-6} \mathrm{~S} / \mathrm{cm}$ respectively. when compared to pure polyaniline there is an increase of one order of conductivity in the metal oxide composite and three order increase of conductivity in the nano metal composite. The result shows that nano metal composite posses better electrical conductivity than pure Pani. This enhanced conductivity of n-Al Pani is due to incorporation of metal particles into the polymer matrix. After doping, the increase in the A.C electrical conductivity of n-Al Pani may be due to the even distribution of nano particles and increase in crystallite density in unit space which is evidence from the XRD result. The combination of amorphous and crystalline structure in the composite material may also be the reason for improved conductivity. ${ }^{17}$

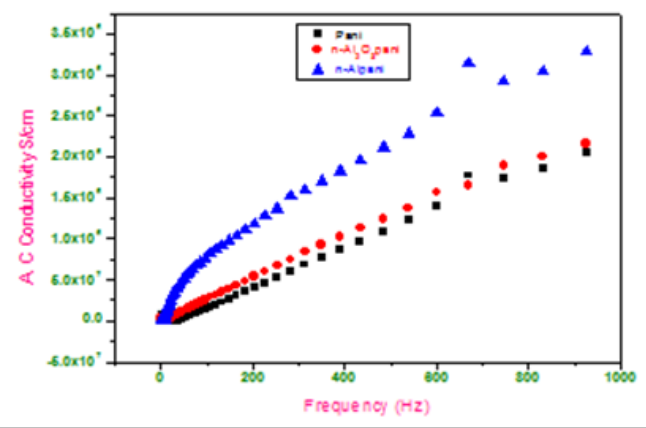

Figure 4 A. C Conductivity of Pure polyaniline, $n-\mathrm{Al}_{2} \mathrm{O}_{3}$ Pani and n-AIPani.

\section{Conclusion}

Pure polyaniline, $\mathrm{n}-\mathrm{Al}_{2} \mathrm{O}_{3}$ Pani and $\mathrm{n}-\mathrm{Al}$ Pani were synthesized by adopting a facile chemical oxidation polymerization method. The structure of the pure pani and its composites has been confirmed by FT-IR study. The average crystalline sizes have been estimated to be around $\mathrm{n}-\mathrm{Al}$ pani and $\mathrm{n}-\mathrm{Al}_{2} \mathrm{O}_{3}$ pani is 31 and $38 \mathrm{~nm}$. The FESEM morphology showed that the Pani and $\mathrm{n}-\mathrm{Al}$ pani and $\mathrm{n}-\mathrm{Al}_{2} \mathrm{O}_{3}$ pani has the morphological modification due to doping and EDX study reveals that the aluminium and aluminium oxide nanocomposites is evenly distributed through the polymer matrix. The increased conductivity was attributed to the formation of a better charge transport network in the relatively insulating PANI matrix. The improvement in the electrical conductivities of these composites is expected to enhance the potential application of the polymer. The results show enhancement in photo degradation of dyes is due to $\mathrm{Al}$ polyaniline crystallite size increase and decrease in particle size, which leads to higher surface and adsorptive nature.

\section{Acknowledgements}

None.

\section{Conflict of interest}

The author declares no conflict of interest.

\section{References}

1. Ameen S, Akhtar MS, Ansari SG, et al. Electrophoretically deposited polyaniline/ZnO nanoparticles for $\mathrm{p}-\mathrm{n}$ heterostructure diodes. Superlattices and Microstructures. 2009;46(6):872-880.

2. Sathiyanarayanan S, Azim SS, Venkatachari G. Preparation of polyaniline- $\mathrm{TiO}_{2}$ composite and its comparative corrosion protection performance with polyaniline. Synthetic Metals. 2007;157(4-5):205-213.

3. Stejskal J, Sapurina I, Trchova M. Polyaniline nanostructures and the role of aniline oligomers in their formation. Progress in Polymer Science. 2010;35(12):1420-1481.

4. Gupta K, Jana PC, Meikap AK. Optical and electrical transport properties of polyaniline-silver nanocom-posite. Synthetic Metals. 2010;160(1314):1566-1573.

5. Umare SS, Shambharkar BH, Ningthoujam RS. Synthesis and characterization of polyaniline- $\mathrm{Fe}_{3} \mathrm{O}_{4}$ nanocomposite: electrical conductivity, magnetic, electro-chemical studies. Synthetic Metals. 2010;160(17-18):1815-1821.

6. Mc Crum NG, Buckley CP, Bucknall CB. Principles of polymer engineering. 2nd ed. New York, USA: Oxford University Press; 1997. $1 \mathrm{p}$. 
7. Dispenza C, Sabatino MA, Chmielewska D, et al. React Funct Polym 2012;72:85-197.

8. Zhong-Ai Hu, Yu-Long Xie, Yao-Xian Wang, et al. Polyaniline/SnO2 nanocomposite for supercapacitor applications. Materials Chemistry and Physics. 2009;114(2-3):990-995.

9. Ashutosh Tiwari, Vandana Singh. Microwave-induced synthesis of electrical conducting gum acacia-graft-polyaniline. Carbohydr Polym. 2008;74:427-434

10. Yadav JB, Patil RB, Puri RK, et al. Studies on spin coated PANI/PMMA composite thin film: effect of post-deposition heating. Appl Surf Sci. 2008;255(5):2825-2829.

11. ChenY, Kang ET, Neoh KG, et al. Covalent immobilization of invert as onto the surface-modified polyaniline from graft copolymerization with acrylic acid. Eur Polym J. 2000;36(10):2095-2103.

12. Colomban $\mathrm{Ph}$. Structure of oxide gels and glasses by infrared and raman scattering. J Mater Sci. 1989;24(8):3002-3010.
13. Paul Mc Millan, Bernard Piriou. The structures and vibrational spectra of crystals and glasses in the silica-alumina system. J Non Cryst Solids. 1982;53(3):279-298.

14. Mazza Daniele, Vallino Mario, Busea Guide. Mullite-type structures in the systems $\mathrm{Al}_{2} \mathrm{O}_{3}-\mathrm{Me}_{2} \mathrm{O}(\mathrm{Me}=\mathrm{Na}, \mathrm{K})$ and $\mathrm{Al}_{2} \mathrm{O}_{3}-\mathrm{B}_{2} \mathrm{O}_{3} . J$ Am Ceram Soc. 1992;75(7):1929-1934.

15. Schnitzler DC, Meruvia MS, Hummelgen IA, et al. Preparation and characterization of novel hybrid materials formed from $(\mathrm{Ti}, \mathrm{Sn}) \mathrm{O}_{2}$ nanoparticles and polyaniline. Chem Mater. 2003;15(24):4658-4665.

16. Huang WS, Humphrey BD, Mac Diarmid AG. Polyaniline, a novel conducting polymer. Morphology and chemistry of its oxidation and reduction in aqueous electrolytes. $J$ Chem soc Faraday Trans. 1986;82(8):2385

17. Straumal BB, Protasova SG, Mazilkin AA, et al. Amorphous interlayers between crystalline grains in ferromagnetic $\mathrm{ZnO}$ films. Mater Lett. 2012;71:21-24. 